\title{
Fractura de Base de Cráneo en Recién Nacidos: Caso Clínico
}

\author{
JUAN CARLOS CASSINA F. ${ }^{1}$, DANIELA QUIROGA M. ${ }^{2}$, PATRICIA MENA N. ${ }^{3}$ \\ 1. Interno, Escuela de Medicina, Pontificia Universidad Católica de Chile. \\ 2. Interna, Escuela de Medicina, Universidad de los Andes. \\ 3. Profesor Asociado Adjunto, Pontificia Universidad Católica de Chile.
}

\begin{abstract}
Basal Fracture of the Skull in a Newborn: Case Report and Review of the Literature

Introduction: Reported incidence of cranial lesions in childbirth is about 1.7 to 59 per 10,000 births. Among these, fracture of the base of the skull is rare but serious due to sequelae and mortality. Objective: Describe a case of a newborn with a fracture of the temporal bone, review diagnosis and management of skull fractures. Clinical Case: The patient was born with assistance, use of forceps, showed deviation of the buccal commisure and otorrhagia on the left side during puerperium. CT Scan detected fracture of the base of the cranium at the level of the temporal bone. Hemogram, cultures and spinal fluid studies were done. Prophylactic therapy was initiated. All blood studies were negative and follow up studies were normal. Discussion: Literature reviews support the diagnosis to be confirmed through a CT scan, and an active search for meningitis be started through hemocultures, hemogram, and Cerebrospinal Fluid. Management should be conservative, evaluating the need for an LP. Evidence shows that antibiotic prophylaxis for meningitis has little indication in these cases.

(Key words: Skull, basal fracture, newborn, meningitis).

Rev Chil Pediatr 2010; 81 (1): 58-63
\end{abstract}

\section{RESUMEN}

Introducción: La incidencia de lesiones de cráneo en recién nacidos reportadas son del orden de 1,7 a 59 por 10000 nacimientos. Dentro de estas, las fracturas de base de cráneo son eventos poco frecuentes, pero de consideración dado su potencial gravedad en cuanto a mortalidad y secuelas. Objetivo: Relatar el caso de un recién nacido con una fractura de peñasco y revisar el diagnóstico y manejo de las lesiones de cráneo. Caso Clínico: El paciente, tras parto instrumentalizado por fórceps, presentó desviación de comisura bucal y otorragia durante su estadía en puerperio. La TAC de cerebro con reconstrucción ósea fue compatible con una fractura de base de cráneo (peñasco izquierdo). Se tomaron hemocultivo, hemograma y PCR y se inició antibioticoterapia profiláctica para meningitis. Evolucionó favorablemente con hemocultivos negativos, hemograma y PCR normales, TAC de control sin cambios y examen neurológico normal. Discusión: La literatura recomienda que el diagnóstico de fractura de base de cráneo debe ser confirmado por medio de TAC de

Trabajo recibido el 08 de junio de 2009, devuelto para corregir el 28 de julio de 2009, segunda versión el 29 de septiembre de 2009, aceptado para publicación el 05 de octubre de 2009.

Correspondencia a:

Juan Carlos Cassina F.

E-mail: jccassin@puc.cl 
cerebro y que debe realizarse una búsqueda activa de meningitis por medio de hemocultivos, hemograma y PCR, evaluar la necesidad de punción lumbar y manejar en forma conservadora. La evidencia señala que la profilaxis antibiótica para meningitis en estos pacientes no tiene indicación.

(Palabras clave: Fractura base de cráneo, recién nacidos, meningitis).

Rev Chil Pediatr 2010; 81 (1): 58-63

\section{Introducción}

Se define trauma obstétrico, como aquellas lesiones que afectan al RN, derivadas del trabajo de parto, asociadas o no a maniobras obstétricas ${ }^{1}$. Diversos estudios muestran que su incidencia tiene una clara relación con el grado de control del embarazo y la atención del trabajo de parto por un equipo médico capacitado, resultando este factor más importante incluso que la vía de parto ${ }^{2}$. Como consecuencia de los diversos avances tecnológicos y la mejora en la práctica obstétrica, la mortalidad por trauma obstétrico ha presentado una clara disminución, estimándose en menos de $4 \mathrm{x}$ 100000 nacidos vivos; sin embargo, los traumatismos no letales son comunes, calculándose una notable mayor incidencia, siendo para lesiones importantes de aproximadamente 2,7 por 1000 nacidos vivos ${ }^{1}$. Las lesiones de cráneo se han reportado con una incidencia de 1,7 a 59 por $10000^{3}$.

El objetivo del presente artículo es comunicar un caso de fractura de base de cráneo en un recién nacido trasladado al Hospital Dr. Sótero del Río y la revisión de la literatura utilizando las bases de datos Pubmed y ScieLO.

\section{Caso Clínico}

Se trata de un recién nacido $(\mathrm{RN})$ de término de 39 semanas, adecuado para la edad gestacional, hijo de madre primigesta de 21 años. Nació tras parto instrumental por forceps, por expulsivo detenido y que presentó una circular reductible al cuello. El recién nacido se encontraba en buenas condiciones generales, destacando al examen físico una discreta desviación de la comisura labial que desapareció espon- táneamente. Se tomaron exámenes de rutina, donde se encontró gases en cordón con $\mathrm{pH}$ de $7,15, \mathrm{pO}_{2}$ de $23,8 \mathrm{mmHg}$, EB de $-18,7$ y Bic de 8,2 . Hto de $66 \%$ y glicemia capilar de 72 $\mathrm{mg} / \mathrm{dl}$.

Se trasladadó a puerperio con su madre, donde se mantuvo en buenas condiciones generales. Sin embargo, en control habitual se pesquisa otorragia de escasa cuantía en conducto auditivo externo izquierdo, por lo que se decide su traslado a la Unidad de Neonatología para su hospitalización y estudio.

Al ingreso en Neonatología se encontraba en buenas condiciones generales, sin otorragia activa. Se repitieron hematocrito, gases venosos y glicemia; los que resultaron normales. Junto a esto se realizó tomografía computada (TC) cerebral con ventana ósea y reconstrucción de peñasco, en el cual no se observó rasgo de fractura, pero sí una ocupación de celdilla izquierda por sobre oído medio con elementos sugerentes de sangre. Dado cuadro compatible con fractura de base de cráneo se inició tratamiento antibiótico empírico con ampicilina y cefotaxima, luego de tomar de hemocultivo y screening infeccioso. Fue trasladado al Hospital Dr. Sótero del Río para evaluación neuroquirúrgica, observándose un examen neurológico normal y ausencia de otorragia activa. Se realizó nuevo TC donde se constató una fractura de peñasco izquierdo, sin signos de otorragia activa (figura 1). Se indicó monitoreo neurológico estricto y TC de control a las 24 horas. Evolucionó favorablemente, afebril, con evaluación neurológica normal y TC de control sin cambios con respecto al anterior. Dada la excelente evolución se suspendieron los antibióticos y se mantuvo en control de signos clínicos. 


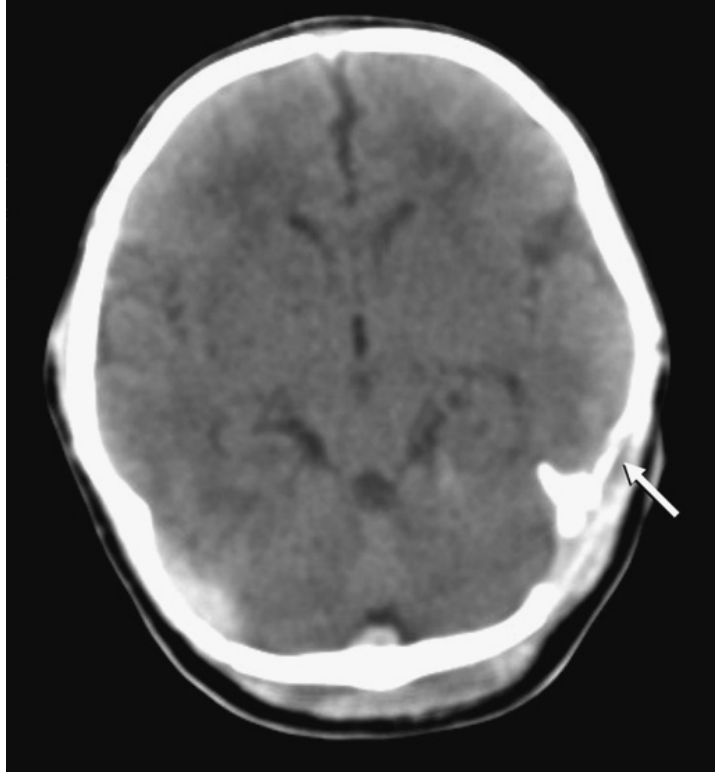

Figura 1. TAC de cerebro con reconstrucción ósea. Flecha muestra la zona de fractura en peñasco izquierdo.

\section{Discusión}

\section{Epidemiología}

La Comisión de acreditación Hospitalaria de Estados Unidos (JCAHO) reportó que en un seguimiento desde el año 1996 al 2004 se pesquizaron 47 casos de muerte neonatal asociadas a traumatismo del parto como causa de muerte. Este mismo estudio señala que el trauma obstétrico ocurre en el 2 a $7 \%$ de los partos y que estos tienen una mayor morbilidad y mortalidad que aquellos no asociados a traumatismo ${ }^{3}$.

Las lesiones de cabeza y cráneo en el recién nacido pueden llegar a tener una frecuencia considerable, reportándose incidencias variables, de hasta 1,7 a 59 por 10000 nacimientos $^{4,5}$. Un estudio multicéntrico realizado en Londres reportó una incidencia de fracturas de cráneo de 3,7 por cada 100000 nacidos vivos, siendo 2,7 veces más frecuente su asociación con partos instrumentalizados, en comparación con aquellos catalogados como espontáneos; en este último grupo se incluyeron partos vía vaginal o cesárea sin uso de fórceps ${ }^{6}$. $\mathrm{Si}$ bien el riesgo de lesiones mayores asociadas (hematoma subdural o subaracnoídeo) es in- frecuente, existen casos reportados, asociados a fractura de cráneo o en ausencia de esta.

\section{Clasificación}

Las fracturas basilares de cráneo (FBC) suelen asociarse en general a mecanismos traumáticos de baja energía y de rasgo lineal. Pueden clasificarse según la región anatómica comprometida, lo que se asocia a su vez a una presentación clínica en particular.

Las fracturas del hueso temporal corresponden al $75 \%$ de las FBC, se subclasifican según el rasgo de fractura en longitudinales, transversas y mixtas ${ }^{7}$ :

Las longitudinales corresponden al 70-90\% de las FBC de hueso temporal, involucran en general la región temporo-parietal, extendiéndose de la porción escamosa del hueso temporal hacia la pared superior del conducto auditivo externo y tímpano, anterior o posteriores a la cóclea y laberinto, terminando en la fosa craneana media cerca o dentro del foramen mastoideo respectivamente.

Las transversales son el 5-30\% de las FBC. Se extienden desde el foramen magno hacia la cóclea y laberinto, terminando en la fosa craneal media.

Las mixtas involucran los otros dos tipos y son las menos frecuentes. También es posible clasificar las FBC de hueso temporal, según comprometan o no el hueso petroso, siendo este último tipo fracturas las no relacionadas con déficit de nervio craneano ${ }^{8}$.

\section{Presentación Clínica}

Dada la ausencia de series clínicas adecuadas en recién nacidos con este tipo de fractura, se describe a continuación las características clínicas presentadas en pacientes pediátricos.

Las FBC que involucran la porción del peñasco pueden presentarse con otorrea y hematomas retroauriculares (signo de Battle). Las FBC de fosa anterior se presentan con rinorrea y hematomas perioculares (signo de ojo de mapache). El compromiso de conciencia y el puntaje de Glasgow asociados, depende del compromiso intracraneano asociado?.

La fractura longitudinal del hueso temporal, puede asociarse a disrupción de la cadena oscicular e hipoacusia de conducción mayor a 
$30 \mathrm{~dB}$, que puede persistir por 6 a 7 semanas. Las hipoacusias que se resuelven en menos de 3 semanas se asocian a hemotímpano y edema de la mucosa del oído medio. Paresia, parestesia facial o nistagmus, son secundarios a compromisos del VII, V y VI par craneano, respectivamente. La fractura transversa de hueso temporal, que involucra el VIII par y laberinto, pueden presentarse con nistagmus, ataxia e hipoacusia neuronal.

\section{Complicaciones}

Las fracturas de cráneo de rasgo lineal pueden asociarse a hematomas extracraneales (cefalohematomas), intracraneales (epidural, subdural) o contusión cerebral. Otra rara complicación corresponde a la formación de quistes leptomeníngeos o fractura de cráneo creciente (growing skull fracture).

Kadish et al, realizaron un estudio retrospectivo de las complicaciones asociadas a FBC en pacientes pediátricos con examen neurológico normal, Glasgow 15 y TC que descartó patología intracraneana, cuadro definido por los autores como FBC simple. De un total de 239 pacientes, 114 (48\%) fueron incluidos en el grupo de FBC simple. De estos los vómitos fueron la complicación más frecuente $(6 \%)$, en el $1 \%$ de los casos se pesquizó meningitis asociada. No hubo casos reportados de hemorragia intracraneal tardía ni necesidad de tratamiento quirúrgico ${ }^{10}$.

\section{Manejo}

Estudio por imágenes: Diversos estudios muestran que la utilización de radiografía como método de estudio es sub-óptimo en revelar fracturas de base de cráneo. Existen ciertas excepciones como lo es la fractura de vertex, que puede no hallarse mediante TC y sí con radiografía simple, pero dada su baja frecuencia no existe un real beneficio cuando se ordena la toma de imágenes. Es por esto que el TC es la modalidad de criterio estándar para el diagnóstico de FBC. La RNM o la angioresonancia son de gran utilidad en la sospecha de daño ligamentoso o lesiones vasculares. Sin embargo, las lesiones óseas son mucho mejor visualizadas usando $\mathrm{TC}^{11}$.

Antibioticoterapia: La FBC predispone al paciente a la posibilidad de diversas infecciones, teniendo gran importancia dentro de estas la meningitis. Lo antes mencionado se debe al contacto con bacterias presentes en los senos paranasales, nasofaringe $u$ oído medio con el sistema nervioso central. La observación de goteo de líquido cerebro espinal (LCE) ha sido asociada con un alto riesgo de contraer meningitis. Es práctica común el uso de antibióticos de manera profiláctica, sin embargo, su rol en la prevención de meningitis no está bien establecida. Diversos estudios buscan descifrar esta interrogante. Un metanálisis realizado por el Dr. M. Retilal ${ }^{12}$, demostró que no existen diferencias significativas con el uso de profilaxis antibiótica respecto a la reducción en la frecuencia de meningitis, las causas de mortalidad, meningitis relacionada a mortalidad y la necesidad de corrección quirúrgica en pacientes con pérdida de LCE. De esta forma la evidencia no apoya el uso de antibiótico profilaxis en pacientes con FBC, exista evidencia de pérdida de líquido cefalorraquídeo o no ${ }^{12-14}$. En el caso del recién nacido además la fractura se produce, generalmente, en un paciente que no está aún colonizado.

\section{Conclusión}

En resumen, la FBC es una complicación muy infrecuente pero potencialmente grave de partos instrumentalizados. La existencia de complicaciones depende, fundamentalmente, del grado de compromiso intracraneano incluyendo las complicaciones de causa infecciosas, en particular, la meningitis asociada. El concepto de FBC simple está definido por aquella que presenta examen neurológico normal y TC que descarte patología traumática intracraneana. Se han reportado una incidencia baja de complicaciones en estos pacientes, sin embargo, es posible encontrar meningitis asociada, de manera que se recomienda manejar conservadoramente a pacientes que cumplen con criterios de FBC simple, una vez descartada la coexistencia de meningitis asociada, luego de una búsqueda activa: condiciones generales del paciente, constatar hiper o hipotermia, hemograma, PCR, hemocultivos y con- 
siderar la necesidad de realizar una punción lumbar. Aquellos casos de FBC simples en los cuales se haya descartado suficientemente la presencia de meningitis, es posible el manejo en una unidad de puerperio hasta el alta materna y control ambulatorio. En los casos de FBC que no cumpla con criterios de FBC simple recomendamos hospitalización al menos en unidad de cuidados intermedios y considerar más sensiblemente la posibilidad de punción lumbar, sobretodo en casos en que exista evidencia de comunicación del medio externo con la cavidad intracraneana, ya sea por drenaje de líquido cefalorraquídeo (otorrea, rinorrea) u otorragia.
En todo recién nacido con el diagnóstico de FBC y se debe realizar otoscopia como parte del examen físico de rutina. Al alta o en el curso de la hospitalización se debe profundizar el estudio de complicaciones auditivas, derivando a otorrinolaringología, en lo posible dentro de 3 a 6 semanas, dado que es el período de corte para lesiones asociadas a disrrupción oscicular versus lesiones edematosas y/o hemorrágicas que son transitorias.

El estudio por imágenes de FBC debe realizarse a través de un TC, salvo sospecha de compromiso de vertex, caso en el cual se debe adicionar al estudio una radiografía de cráneo.

No existe evidencia que sustente el uso de

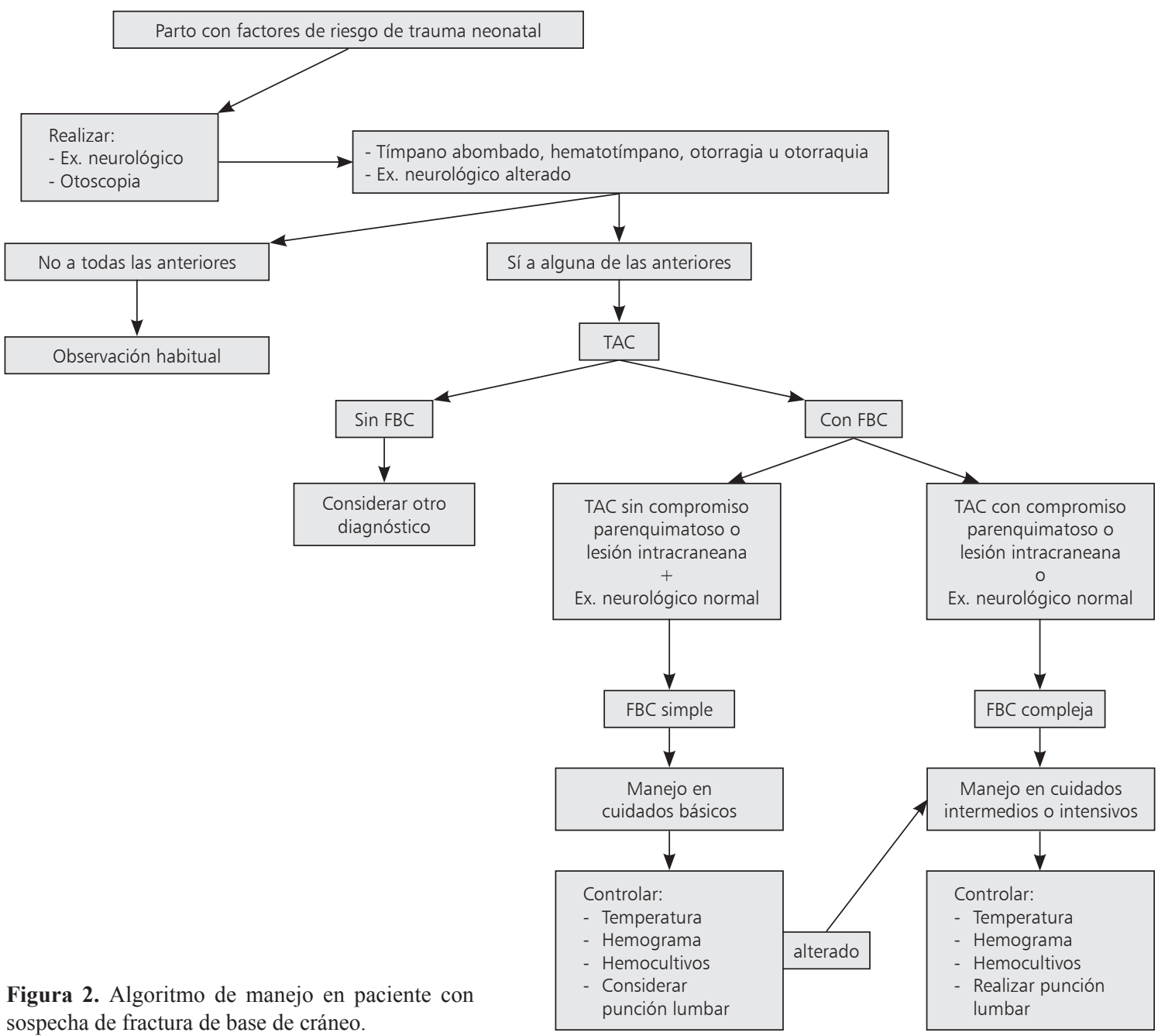


antibioterapia profiláctica en estos pacientes, presenten o no pérdida de LCR al exterior.

Proponemos un algoritmo para el manejo de recién nacidos con sospecha de fractura de base de cráneo (figura 2).

\section{Referencias}

1.- Mena P, Mühlhausen G, Novoa J, et al: Traumatismos del parto. En: Guías Nacionales de Neonatología, Ministerio de Salud de Chile, Santiago 2005; 415-20.

2.- Rouse D, Owen J: Prophylactic cesarean delivery for fetal macrosomia diagnosed by means of ultrasonography. A Faustian bargain? Am J Obstet Gynecol 1999; 181: 332-8.

3.- Parker L: Part 1: Early recognition and treatment of birth trauma: injuries to the head and face. Adv Neonatal Care 2005; 5: 288-97.

4.- Amar A, Aryan H, Meltzer H, Levy M: Neonatal subgaleal hematoma causing brain compression: report of two cases and review of the literature. Neurosurgery 2003; 52: 1470-4.

5.- Kilani R, Wetmore J: Neonatal subgaleal hematoma: presentation and outcome -radiological findings and factors associated with mortality. Am J Perinatol 2006; 23: 41-8.

6.- Dupuis O, Silveira R: Comparasion of "instrumentassociated" and "spontaneous" obstetric depressed skull fractures in a cohort of 68 neonates. Am J Gynecol Obstet 2005; 192: 165-70.

7.- Wennmo C, Spandow O: Fractures of the temporal bone: chain incongruencies. Am J Otolaryngol 1993; 14: 38 42.

8.- Ishman S, Friedland D: Temporal bone fractures: traditional classification and clinical relevance. Laryngoscope 2004; 114: 1734-41.

9.- Anderson P, Montesano P: Morphology and treatment of occipital condyle fractures. Spine 1988; 13: 731-6.

10.- Kadish H, Schunk J: Pediatric basilar skull fracture: do children with normal neurologic findings and no intracranial injury require hospitalization? Ann Emerg Med 1995; 26: 37-41.

11.- Legros B, Fournier P, Chiaroni P, et al: Basal fracture of the skull and lower (IX, X, XI, XII) cranial nerves palsy: four case reports including two fractures of the occipital condyle, a literature review. J Trauma 2000; 48: 342-8.

12.- Ratilal B, Costa J, Sampaio C: Antibiotic prophylaxis for preventing meningitis in patients with basilar skull fractures. Cochrane Database Syst Rev 2006; 25;(1): CD004884.

13.- Villalobos T, Arango C, Kubilis P, Rathmore M: Antibiotic prophylaxis after basilar skull fractures: a metaanalysis. Clin Infect Dis 1998; 27: 364-9.

14.- Al-Haddad S, Kirollos R: A 5-year study of the outcome of surgically treated depressed skull fractures. Ann R Coll Surg Engl 2002; 84: 196-200. 